\title{
BMJ Open BOND study: a randomised double-blind, placebo-controlled trial over 12 months to assess the effects of benfotiamine on morphometric, neurophysiological and clinical measures in patients with type 2 diabetes with symptomatic polyneuropathy
}

Gidon J Bönhof (1D , ${ }^{1,2}$ Gundega Sipola, ${ }^{1}$ Alexander Strom, ${ }^{1,3}$ Christian Herder, ${ }^{1,2}$
Klaus Strassburger, ${ }^{3,4}$ Birgit Knebel, ${ }^{3,5}$ Claudia Reule, ${ }^{6}$ Jan-Christoph Wollmann, Andrea Icks, ${ }^{7,8}$ Hadi Al-Hasani, ${ }^{3,5}$ Michael Roden, ${ }^{1,2}$ Oliver Kuss, ${ }^{4,9}$ Dan Ziegler ${ }^{1,2}$
To cite: Bönhof GJ, Sipola G, Strom A, et al. BOND study: a randomised double-blind, placebo-controlled trial over 12 months to assess the effects of benfotiamine on morphometric, neurophysiological and clinical measures in patients with type 2 diabetes with symptomatic polyneuropathy. BMJ Open 2022;12:e057142. doi:10.1136/ bmjopen-2021-057142

- Prepublication history and additional supplemental material for this paper are available online. To view these files, please visit the journal online (http://dx.doi.org/10.1136/ bmjopen-2021-057142)

GJB and GS contributed equally.

Received 07 September 2021 Accepted 05 January 2022

Check for updates

(c) Author(s) (or their employer(s)) 2022. Re-use permitted under CC BY-NC. No commercial re-use. See rights and permissions. Published by BMJ.

For numbered affiliations see end of article.

Correspondence to Professor Dan Ziegler; dan.ziegler@ddz.de

\section{ABSTRACT}

Introduction Diabetic sensorimotor polyneuropathy (DSPN) affects approximately $30 \%$ of people with diabetes, while around half of cases are symptomatic. Currently, there are only few pathogenetically oriented pharmacotherapies for DSPN, one of which is benfotiamine, a prodrug of thiamine with a high bioavailability and favourable safety profile. While benfotiamine has shown positive effects in preclinical and short-term clinical studies, no long-term clinical trials are available to demonstrate disease-modifying effects on DSPN using a comprehensive set of disease-related endpoints.

Methods and analysis The benfotiamine on morphometric, neurophysiological and clinical measures in patients with type 2 diabetes trial is a randomised doubleblind, placebo-controlled parallel group monocentric phase II clinical trial to assess the effects of treatment with benfotiamine compared with placebo in participants with type 2 diabetes and mild to moderate symptomatic DSPN. Sixty participants will be 1:1 randomised to treatment with benfotiamine $300 \mathrm{mg}$ or placebo two times a day over 12 months. The primary endpoint will be the change in corneal nerve fibre length assessed by corneal confocal microscopy (CCM) after 12 months of benfotiamine treatment compared with placebo. Secondary endpoints will include other CCM measures, skin biopsy and function indices, variables from somatic and autonomic nerve function tests, clinical examination and questionnaires, general health, health-related quality of life, cost, safety and blood tests.

Ethics and dissemination The trial was approved by the competent authority and the local independent ethics committee. Trial results will be published in peer-reviewed journals, conference abstracts, and via online and print media.

Trial registration number DRKS00014832.

\section{Strengths and limitations of this study}

The benfotiamine on morphometric, neurophysiological and clinical measures in patients with type 2 diabetes study is the first randomised, doubleblind, placebo-controlled clinical trial to assess the long-term effects of benfotiamine in type 2 diabetes individuals with symptomatic diabetic sensorimotor polyneuropathy (DSPN) over 12 months.

- A highly comprehensive array of morphometric, functional and clinical endpoints derived from pathogenetic pathways implicated in the development of DSPN was included to evaluate the diseasemodifying potential of benfotiamine on different components of DSPN.

- While the rationale for focusing on mild to moderate DSPN was to increase the probability that less severe disease stages could be more susceptible to an intervention than severe DSPN, a positive trial outcome would not be generalisable to the latter.

\section{INTRODUCTION}

Diabetic sensorimotor polyneuropathy (DSPN) affects approximately $30 \%$ of people with diabetes mellitus with an incidence of around $2 \%$ per year. DSPN has been defined as a symmetrical, length-dependent sensorimotor polyneuropathy attributable to metabolic and microvascular alterations due to diabetes mellitus and other cardiovascular risk factors. ${ }^{1}$ DSPN is associated with considerable morbidity, increased mortality, foot ulcers and markedly impaired quality of life by causing neuropathic pain and sleep deprivation. ${ }^{2}$ Neuropathic symptoms include 
particularly pain, paraesthesia and numbness, while neuropathic signs comprise sensory deficits to touch, pressure, temperature and pain stimuli. ${ }^{23}$ However, DSPN frequently remains an underdiagnosed and undertreated condition as therapy options are limited. ${ }^{4}$

Current established options for the management of DSPN are mainly based on control of risk factors such as hyperglycaemia and symptomatic treatment to alleviate neuropathic pain. ${ }^{5}$ Near-normal glycaemic control is crucial to delay the development of DSPN in people with type 1 diabetes, although it may not be sufficient to prevent or reverse DSPN. By contrast, there is currently no compelling evidence of favourable effects on the development and progression of DSPN in type 2 diabetes. ${ }^{45}$ Analgesic pharmacotherapy is purely symptomatic, and each individual agent helps to relieve neuropathic pain to a clinically meaningful degree only in around half of the patients. Moreover, it is unclear whether analgesic compounds may also alleviate non-painful symptoms such as paraesthesia or numbness, which are frequently reported by patients with DSPN. ${ }^{4}$

The available evidence for the effectiveness of pathogenetically oriented disease-modifying pharmacotherapy is still limited. ${ }^{4}$ Currently only three agents derived from the pathophysiological pathways implicated in the development and progression of DSPN are available in several countries: $\alpha$-lipoic acid, benfotiamine and actovegin, whereas other agents such as aldose reductase inhibitors, nerve growth factor, protein kinase $\mathrm{C}$ (PKC) $B$ inhibitor, long-acting (PEGylated) C-peptide or vitamin E were not effective in DSPN. ${ }^{4}$

\section{Benfotiamine}

Benfotiamine is a lipid-soluble derivate of thiamine (vitamin $\mathrm{B}_{1}$ ) with a high bioavailability. Compared with the water-soluble thiamine, it is passively absorbed, passes the intestinal barrier faster and accomplishes higher blood concentrations. ${ }^{4}$ In experimental diabetes models, benfotiamine was demonstrated to act on several major pathways implicated in the pathogenesis of diabetic microvascular complications (polyol pathway, hexosamine pathway, advanced glycation end product (AGE) formation pathway, and diacylglycerol-PKC pathway), which are activated by increased availability of the glycolytic metabolites glyceraldehyde-3-phosphate and fructose-6phosphate. ${ }^{6} 7$ Furthermore, benfotiamine exerts direct antioxidant properties. $^{8} 9$ In experimental diabetic neuropathy, benfotiamine-but not thiamine-treatment nearly normalised nerve conduction velocity (NCV), induced an inhibition of neural imidazole-type AGE formation, and completely prevented diabetes-induced glycoxidation products after 6 months ${ }^{10}$ suggesting that benfotiamine rather than thiamine could be a suitable candidate drug for pathogenetically oriented diseasemodifying pharmacotherapy of DSPN. ${ }^{10}$

Several studies conducted over periods of up to 12 weeks demonstrated that benfotiamine alone or in combination with vitamin $\mathrm{B}_{6}$ and $\mathrm{B}_{12}$ alleviates symptoms and deficits of DSPN. ${ }^{11-13}$ In contrast, no differences in changes in nerve conduction variables were observed in a recent study including individuals with type 1 diabetes after 24 months of treatment with benfotiamine $300 \mathrm{mg} /$ day compared with placebo. ${ }^{14}$ However, participants had almost normal nerve function prior to treatment which did not leave any room for a meaningful degree of improvement. ${ }^{15}$ In a recent pilot randomised controlled trial (RCT) which was terminated prematurely due to technical reasons, administration of benfotiamine over 6 months $(600 \mathrm{mg} /$ day for 3 months, followed by $300 \mathrm{mg} /$ day until the study end) resulted in a reduction of neuropathic symptoms assessed by the Michigan Neuropathy Screening Instrument (MNSI) questionnaire, while changes in neuropathic deficits (MNSI physical examination), quality of life and pain did not reach statistical significance. ${ }^{416}$ In people with diabetes as well as in tobacco smoking individuals, benfotiamine was shown to improve endothelial function. ${ }^{17} 18$ The safety profile of benfotiamine is excellent. ${ }^{4}$ Milgamma mono 300 (containing $300 \mathrm{mg}$ benfotiamine) was approved in 2005 for the treatment or prophylaxis of clinically manifest vitamin $\mathrm{B} 1$ deficiency if a dietary supplementation is not effective ${ }^{19}$ and for the treatment of neuropathies and cardiovascular disorders caused by vitamin $\mathrm{B}_{1}$ deficiency.

\section{Rationale}

Based on preclinical and clinical data, the use of benfotiamine could prevent or limit the progression of DSPN. Except for one short-term trial over 12 weeks demonstrating that benfotiamine in combination with vitamin $\mathrm{B}_{6}$ and $\mathrm{B}_{12}$ may improve $\mathrm{NCV},{ }^{11}$ no longer-term clinical study specifically in people with type 2 diabetes and DSPN is available to demonstrate whether benfotiamine may favourably modify the development and progression of DSPN rather than alleviate neuropathic symptoms only. Thus, there is an unmet need for further studies with benfotiamine. Whether benfotiamine can be considered a disease-modifying treatment of DSPN based on pathogenetic considerations can only be answered by well-designed long-term ( $\geq 1$ year) RCTs.

Since it remains unclear which components of DSPN may be most susceptible to benfotiamine treatment, the present exploratory proof-of-concept (POC) study includes an array of clinical, functional and morphological outcomes with emphasis on small fibre neuropathy. The benfotiamine on morphometric, neurophysiological and clinical measures in patients with type 2 diabetes (BOND) study will assess the efficacy and safety of benfotiamine over 1 year to determine whether this drug could represent a disease-modifying therapy approach with beneficial effects on clinical and selfreported outcomes as well as morphological and functional parameters of DSPN. Benfotiamine is expected to be well tolerated, and altogether represents a suitable candidate to address an important public health issue.

\section{METHODS AND ANALYSIS}

\section{Trial design}

The BOND study is a randomised, double-blind, placebocontrolled, parallel group POC phase II trial to assess 
the effects of treatment with benfotiamine for 12 months on morphometric, neurophysiological, clinical and health economic measures in 60 participants with type 2 diabetes and mild to moderate symptomatic DSPN. It is conducted as a monocenter trial at the Institute for Clinical Diabetology, German Diabetes Center at Heinrich Heine University in Düsseldorf, Germany. The sponsor of the trial is Wörwag Pharma GmbH \& Co. KG, Böblingen, Germany. After screening for eligibility, participants will be 1:1 randomly allocated into two groups (30 participants per arm). Recruitment started on 8 October 2018 and is expected to continue until mid-2022. The end of the trial is defined as the last visit of the last randomised participant in the trial. The actual protocol version is 3.0, dated 20 January 2021.

\section{Patient and public involvement}

Patients or the public were not involved in the design, or conduct, or reporting, or dissemination plans of this trial.

\section{Trial sequence}

Potential candidates will be contacted by the trial site to discuss the trial and their willingness to participate. Subsequently, an appointment will be scheduled for obtaining informed consent (online supplemental files 1,2) prior to checking for eligibility and performing the screening assessments. If the participant is eligible, a second visit within 30 days is scheduled for remaining baseline assessments, randomisation and study medication administration. Two weeks after first treatment, participants will have a telephone visit for safety assessment including assessment adverse events and neuropathic symptoms, which will be repeated 3 months after first treatment. After 6 months of treatment, all baseline assessments will be repeated, except for skin biopsy, and new study medication will be dispensed. At 9 months, participants will have a telephone visit to assess safety. At 12 months, all baseline assessments will be repeated, and treatment will be terminated. Two weeks later, a telephone visit will take place for follow-up. Table 1 shows the schedule for the visits and procedures performed during the trial.

Participants may withdraw at any time and for any reason. Participants may be withdrawn from the trial at the discretion of the investigator due to a safety concern or if judged non-compliant with trial procedures.

\section{Recruitment}

Participants are recruited through adverts on social media, leaflets for medical institutions within reasonable distance to the trial site, and from other study cohorts from the German Diabetes Center.

\section{Eligibility criteria}

Individuals with type 2 diabetes and mild to moderate symptomatic DSPN will be eligible. Disorders that might interfere with neurological assessment including neuropathies of any cause other than diabetes (eg, vitamin $B_{12}$ deficiency, chemotherapy induced peripheral neuropathy, or alcoholic polyneuropathy) will be excluded. Complete eligibility criteria are listed in box 1 .

\section{Intervention}

For each randomised participant, the treatment period will be 12 months. As the investigational medicinal product (IMP), one group will receive benfotiamine $300 \mathrm{mg}$ two times a day $(600 \mathrm{mg} /$ day $)$, while the other group will receive placebo. The placebo tablets will have the same appearance and will not be distinguishable from those containing benfotiamine. All participants will be instructed to take one tablet in the morning and one in the evening unchewed with liquid take (before meals).

\section{Randomisation and blinding}

Participants will be randomly assigned to either benfotiamine or placebo in 1:1 ratio by assigning the randomisation number in consecutive order. The IMP contains the randomisation number as given by the computergenerated randomisation code. Products will be provided in a blinded manner along with sealed emergency envelopes for unblinding single participants if necessary. The randomisation list will be kept strictly confidential, and the results of thiamine serum levels will remain at an external laboratory site only, to avoid unintended unblinding. Thiamine results will be included in the database after database lock. An independent statistician and unblinded manufacturer will keep treatment allocation information confidential until database lock. All people involved in the conduct of the trial (including trial site and sponsor and the statistician who performs the analysis in the end), as well as the participants are kept blinded for the whole duration of the trial conduct.

\section{Concomitant and rescue medication}

Any additional drugs considered necessary for the participant's welfare may be given by the attending physicians and at the discretion of the investigator. Treatment that may exert beneficial effects on DSPN and/or neuropathic pain will be excluded, except for a monotherapy with gabapentin, pregabalin or duloxetine without relevant dose change during the trial. Acetaminophen (paracetamol) up to $3 \mathrm{~g}$ /day or metamizole up to $4 \mathrm{~g}$ /day will be permitted as analgesic rescue medication. If rescue medication will be taken on 5 days a week or longer, an interim safety visit will be scheduled.

\section{Outcome measures}

The trial objective is to assess the effects of treatment with benfotiamine compared with placebo over 12 months on morphometric, neurophysiological, clinical and health economic measures in individuals with type 2 diabetes and mild to moderate symptomatic DSPN. The primary objective is the effect on structural measures of small nerve fibre damage, while secondary objectives include the effects on peripheral nerve fibre regeneration, peripheral and autonomic nerve function tests, skin function tests, inflammation, gene expression, mitochondrial 


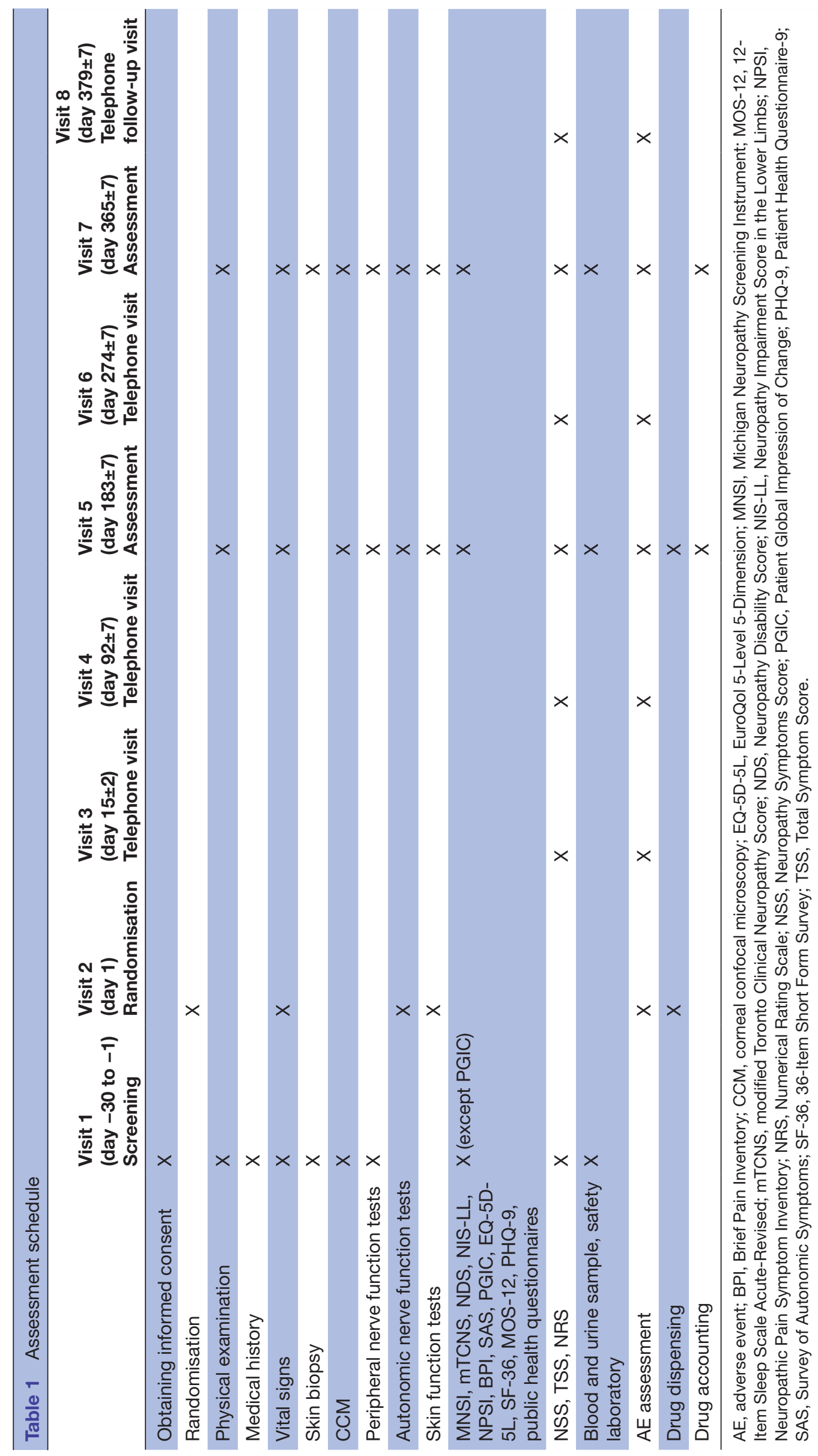

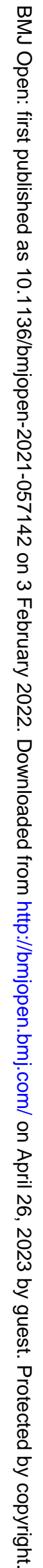




\section{Box 1 Eligibility criteria}

\section{Inclusion criteria}

- Informed consent signed and dated.

- Diabetes mellitus type 2 according to the American Diabetes Association criteria (2017), lasting $\geq 1$ year.

- Stable diabetes metabolism, defined as no metabolic decompensation within the last 3 months (severe hypoglycaemia with unconsciousness, ketoacidosis).

- According to the investigator's opinion no further optimising potential in diabetic control.

- Age: $\geq 18$ years.

- Neuropathic symptoms $\geq 6$ months.

- Presence of mild to moderate diabetic sensorimotor polyneuropathy with Neuropathy Disability Score 3-8 points confirmed by at least one of the following: reduced sural sensory nerve conduction velocity (SNCV), sural sensory nerve action potential), peroneal motor NCV (MNCV), tibial MNCV.

- Measurable sural SNCV, peroneal MNCV or tibial MNCV above detection limit.

- Corneal nerve fibre length $<1$ SD below the mean of control subjects.

- At least one palpable pulse of tibialis posterior artery or dorsalis pedis artery on each side of the foot.

- Stable diabetes medication without optimising potential.

- Stable insulin dose for insulin-dependent patients within the last 3 months prior to screening.

- HbA1c $<9.5 \%$.

- Acceptable contraceptive measures with female subjects in childbearing potential.

- Ability and willingness to abstain from alcohol and from engaging in strenuous physical activity from 24 hours prior to each visit until discharge from the unit.

- Ability to meet the study centre visits for the study duration.

\section{Exclusion criteria}

- Subjects with secondary forms of diabetes such as due to pancreatitis

- Contraindications, known allergy or hypersensitivity to benfotiamine or other ingredients of the study medication or local anaesthetics.

- Neuropathy of any cause other than diabetes which might interfere with neurological assessment.

- Severe pain other than of neuropathic origin that might impair the assessment of neuropathic pain.

- Diseases causing mixed pain that might interfere with neurological assessment.

- Pain level $>9$ over 24 hours on a numerical 11-point rating scale.

- Proximal asymmetric neuropathy or neuropathic symptoms of the trunk or proximal lower limbs.

- Active foot ulcer.

- Currently active or history of alcohol abuse (defined as an intake of more than 24 units of alcohol per week; one unit of alcohol equals approximately $250 \mathrm{~mL}$ of beer, $100 \mathrm{~mL}$ of wine or $35 \mathrm{~mL}$ of spirits) or drug addiction (including soft drugs like cannabis products).

- Known peripheral arterial occlusive disease Fontaine stage II-IV or no palpable pulse of tibialis posterior artery or dorsalis pedis artery on each side of the foot.

- Neoplasms, except for basal cell carcinoma (basalioma).

- Chronic kidney disease with severely decreased estimated glomerular filtration rate $<30 \mathrm{~mL} / \mathrm{min} / 1.73 \mathrm{~m}^{2}$ at screening using the simplified modification of diet in renal disease equation.

Continued
Box 1 Continued

- As judged by the investigator, serious and/or unstable coronary heart disease (unstable angina, myocardial infarction within 6 months prior to screening), congestive heart failure of New York Heart Association Class III or worse, uncontrolled hypertension, history of congenital QT-syndrome within family, history of stroke (within 6 months prior to screening).

- Bleeding diathesis or treatment with antithrombotic agents (coumarins, heparin, factor $X$ inhibitors, thrombin inhibitors).

- Uncontrolled high blood pressure (diastolic blood pressure $>95 \mathrm{~mm}$ $\mathrm{Hg}$ and/or systolic blood pressure $>160 \mathrm{~mm} \mathrm{Hg}$ ) at screening, unless clearly documented to be white-coat hypertension.

- Clinical or laboratory evidence of hepatic dysfunction or disease; laboratory evidence defined as any of the following parameters: alkaline phosphatase, alanin transaminase (ALT), aspartate transaminase (AST) or bilirubin $>3 \times$ upper limit of normal, except for a mild rise in bilirubin considered to be due to Gilbert's condition.

- Generalised immune diseases (eg, HIV-positive, autoimmune diseases, connective tissue diseases).

- Untreated endocrine diseases like hyperthyroidism or hypothyroidism.

- Treatment, lasting at least 5 days days or longer, with alpha-lipoic acid, B-vitamins, evening primrose oil or deproteinised haemoderivates of calf blood, containing low-molecular-weight compounds of up to $5.000 \mathrm{Da}$ or with other substances with interaction to the study product (eg, 5-fluorouracil) or affecting study endpoints within the last 3 months before screening, except for daily intake of B-vitamins amounting to less than $1500 \%$ of the recommended daily allowance lasting until 1 month prior to screening.

Treatment with cutaneous electrical nerve stimulation, muscle stimulation or acupuncture within the last month before screening.

- Treatment of neuropathic pain with antidepressants, anticonvulsants, sodium channel blockers, opioids, neuroleptics and capsaicin $8 \%$ patch within the last 3 months prior to screening, except for a monotherapy with gabapentin, pregabalin or duloxetine without relevant dose change within the last 2 months prior to screening and during the study.

- Mental, psychiatric or other conditions compromising data collection and understanding of written or oral instructions during the study.

- Pregnant women or nursing mothers.

- Participation in another clinical trial study within the last 3 months prior to screening.

- Blood donation within the last 3 months before screening or during the study.

respiration, clinical examination and questionnaires, general health and economic evaluation.

\section{Primary endpoint}

The primary endpoint will be the difference between the change over 12 months in corneal nerve fibre length (CNFL) determined using corneal confocal microscopy (CCM) in the benfotiamine group and the placebo group. CCM is currently the most suitable non-invasive instrument to assess early changes in small nerve fibre morphology in DSPN trials. ${ }^{20}{ }^{21} \mathrm{~A}$ recent meta-analysis confirmed a reduction of CCM indices in patients with DSPN compared with individuals without DSPN. ${ }^{22}$ Using CCM, several studies have demonstrated that corneal 
nerve fibre loss is associated with increasing severity of DSPN and may precede the development of clinically manifest DSPN. ${ }^{123}$ Improvement in CCM measures were reported after 1 year in participants with type 1 diabetes and severe DSPN who underwent combined pancreaskidney transplantation, suggesting that small nerve fibre repair may be detected with this technique even in patients with advanced nerve damage. ${ }^{24}$ Furthermore, we recently described enhanced corneal nerve branching in individuals with painful DSPN compared with painless DSPN. ${ }^{25}$

\section{Secondary endpoints}

\section{Morphometric assessment}

Secondary morphometric endpoints will include other CCM indices: corneal nerve fibre density (CNFD), main corneal nerve branch density and corneal nerve fibre tortuosity, as well as skin biopsy markers. Skin biopsy is a minimally invasive method that allows for a direct quantification of intraepidermal nerve fibre density (IENFD) in the lower limbs. ${ }^{26}{ }^{27}$ Thus, assessment of IENFD is considered the gold standard for the diagnosis of small fibre neuropathy. ${ }^{28}$ However, only data from uncontrolled studies are available that demonstrated interventionrelated improvements in IENFD in DSPN. ${ }^{29-31}$

Novel biomarkers obtained from skin biopsies have been validated focusing on those targeting microvascular, neurotrophic and inflammatory processes (CD31 for endothelial cells, superoxide dismutase (SOD2) for oxidative stress, GAP-43 and GAP-43/PGP9.5 ratio for nerve regeneration, and langerin (CD207) for epidermal Langerhans cells ${ }^{4}$ as well as posttranslationally modified proteins: protein carbonyls, HNE- nitrotyrosine-modified proteins and AGEs (argpyrimidine, Ne-carboxymethyl lysine, carboxyethyl lysine and methylglyoxal hydroimidazolone).$^{32}$ Recent studies showed that the cutaneous expression of these markers is altered in recent-onset type 2 diabetes in addition to reduced IENFD. ${ }^{33-36}$ Therefore, these markers could be useful in POC studies to demonstrate efficacy of interventions on microvascular, neurotrophic, oxidative or inflammatory processes in patients with DSPN. ${ }^{1}$

\section{Nerve function tests}

Nerve conduction studies (NCSs) are the current gold standard to assess large nerve fibre function and are required to confirm the diagnosis of DSPN. Furthermore, they allow to estimate the severity of the disease. ${ }^{28}$ In the present trial, NCS will be conducted in peripheral nerves of the upper and lower extremities (motor NCV: median, ulnar, tibial, peroneal nerves; sensory NCV and sensory nerve action potential: median, ulnar, sural nerves) using surface electrodes (Neurowerk EMG, Sigma MedizinTechnik, Gelenau, Germany).

Quantitative sensory testing is a suitable tool to noninvasively assess and monitor sensory nerve function in response to controlled stimuli (small fibre function: cold, warmth, pain; large fibre function: vibration)..$^{34}$
Temperature detection thresholds will be determined at the thenar eminence and dorsum of the foot (TSA-II NeuroSensory Analyzer, Medoc, Ramat Yishai, Israel), while vibration perception thresholds (VPTs) will be assessed at the second metacarpal bone and medial malleolus (Vibrameter, Somedic, Stockholm, Sweden). Sensory nerve function bedside tests will include the NeuroQuick (Schweers, Meerbusch, Germany), Vibratip (McCallan Medical, Derby, UK) and ETF $^{128}$ electronic tuning fork (O'Brien Medical, Orono, Maine, USA).

A battery of cardiovascular autonomic reflex tests will be performed to assess cardiovascular autonomic function and to diagnose diabetic cardiovascular autonomic neuropathy, which is characterised by reduced heart rate variability $(\mathrm{HRV}) .{ }^{37}$ These tests include measures at rest: coefficient of variation (CV), spectral analysis of HRV in the very low frequency (LF), LF, high frequency (HF) bands and LF/HF ratio in the supine and standing postures over 5 min each; measures during deep breathing with six breaths/min: CVb, expiration-inspiration ratio, SD; Valsalva ratio and $\max / \min 30: 15$ ratio; and the change in systolic and diastolic blood pressure in response to standing up from the supine position (VariaCardio TF5, MIE, Leeds, UK; Carescape V100 Monitor, GE Healthcare, Solingen, Germany). Moreover, noninvasive measurement of spontaneous baroreflex sensitivity (BRS) will be used to quantify baroreflex regulation of the cardiovascular system (Finometer MIDI, Finapres Medical Systems, Enschede, the Netherlands; Nevrokard BRS Analysis V.6.3.0, Nevrokard, Izola, Slovenia). ${ }^{48}$

The pupillary diameter and light reflex responses will be determined to assess autonomic nerve dysfunction of the pupillomotor system including pupil size, light reflex amplitude, latency, constriction and redilatation velocities (CIP, AMTech, Dossenheim, Germany).

Secretory sweat gland function (sudomotor function) which can be affected by nerve fibre damage due to diabetes, leading to dry skin and higher risk of diabetic foot ulcers, will be assessed by calculation of electrochemical skin conductance at both palms and soles (SUDOSCAN, Impeto Medical, Paris, France) as well as by using a moisture-dependent adhesive indicator test (neuropad, TRIGOcare International, Wiehl, Germany) at the plantar aspect of the foot (analysed both visually and quantitatively).

\section{Skin function tests}

Cutaneous autofluorescence will be assessed noninvasively (AGE Reader, Diagnoptics Technologies, Groningen, the Netherlands) to determine the content of AGEs, a result of carbonyl stress implicated in the pathogenesis of microvascular diabetic complications, as benfotiamine was suggested to reduce AGE formation in the presence of hyperglycaemia. ${ }^{1}$

Lightguide tissue spectrophotometry, a combination of laser Doppler spectroscopy and tissue spectrometry, will be used to evaluate tissue oxygen supply. ${ }^{39}$ Cutaneous blood flow will be measured continuously at the right 
dorsal thenar eminence (O2C, LEA Medizintechnik, Heuchelheim, Germany). After a steady flux is recorded for 3-5 min, the brachial artery is occluded using a pressure cuff placed around the forearm that is inflated to $20 \mathrm{~mm} \mathrm{Hg}$ above systolic blood pressure, held for $5 \mathrm{~min}$ and then deflated. The flux recording is then continued for at least $5 \mathrm{~min}$. Measurements will be carried out in superficial and deep layers as measures of skin and muscle blood flow and oxygenation. ${ }^{39} 40$

\section{Examination and questionnaires}

To assess signs and symptoms of DSPN, ${ }^{4}$ several scores will be used: Neuropathy Disability Score, MNSI physical examination part, Neuropathy Impairment Score-Lower Limbs sensory function and reflexes subscore, modified Toronto Clinical Neuropathy Score, ${ }^{41}$ Neuropathy Symptoms Score, Total Symptom Score, Neuropathic Pain Symptom Inventory (NPSI), 11-point Numerical Pain Rating Scale at day, night and over 24 hours, Brief Pain Inventory short form, 12-Item Sleep Scale AcuteRevised (MOS-12), and Survey of Autonomic Symptoms. Questionnaires for general health, health-related quality of life, depression, safety, tolerability and healthcare use for health economic evaluation include the Patient Global Impression of Change (PGIC), EuroQol 5-Level 5-Dimension questionnaire (EQ-5D-5L), 36-Item Short Form Survey V.1.0, Patient Health Questionnaire-9, a questionnaire on health-related resource use and expenditure, and a tolerability questionnaire.

\section{Inflammation, gene expression, mitochondrial respiration}

Proinflammatory and anti-inflammatory cytokines, chemokines, angiogenesis and growth factors, and soluble adhesion molecules will be measured to assess the effects of benfotiamine on subclinical inflammation. ${ }^{42} 43$

Transcriptome-wide assessment of gene expression in whole blood will be performed to analyse gene expression patterns associated with the prevalence and incidence of DSPN. Moreover, analyses will include transketolase single nucleotide polymorphisms. ${ }^{44}$

Mitochondrial oxidative capacity and oxidative stress will be analysed by measuring the oxygen flux and hydrogen peroxide emission from the peripheral mononuclear blood cells (Oxygraph-2k, Oroboros Instruments, Innsbruck, Austria). ${ }^{45}$ Citrate synthase activity will be assessed as a surrogate marker for mitochondrial content. ${ }^{46}$ Furthermore, overall oxidation-reduction potential and components of antioxidant defence will be measured in serum including assessment of the activity of SOD3. ${ }^{47}$

\section{Measures of pharmacokinetics and safety}

Changes from baseline in thiamine monophosphate and diphosphate, free and total thiamine levels, and thiamine pyrophosphate effect will be assessed for pharmacokinetic characterisation. The occurrence of adverse events and changes in laboratory safety variables and clinical measures (physical examination, vital signs) will be analysed.

\section{Statistical considerations}

Sample size estimation

Sample size calculation is based on CCM, based on a prospective observational study, ${ }^{48}$ since no RCT was available at the time of completing the trial design. In 15 individuals with diabetes, after 12 months following simultaneous pancreas and kidney transplantation, CNFL increased by $4.8524 \pm 5.71388$ (mean $\pm \mathrm{SD}) \mathrm{mm} / \mathrm{mm}^{2}$ and CNFD increased by $5.4536 \pm 6.0545$ fibres $/ \mathrm{mm}^{2}$. A conservative assumption is, that CNFD and CNFL remain unchanged during placebo treatment after 12 months. With a power of 0.80 and two-sided $\alpha=0.05$, the number of participants required for CNFL is 23 per arm (46 in total) and for CNFD 21 per arm (42 in total). Assuming an overall drop-out rate of 20\%, 28 participants per arm (56 in total) were initially planned to be enrolled. Up to four drop-outs were planned to be replaced. Since four randomised participants dropped out already, the number of randomised participants were increased to 30 per arm in the latest amendment.

\section{Statistical analysis}

Differences of baseline to post-treatment changes after 12 months between treatment arms will be analysed by means of analysis of covariance, where the post-treatment outcome enters the model as the dependent variable and a treatment indicator and the baseline outcome, age, sex, COVID-19 information (pre, during and post-COVID-19 pandemic) and parallel treatment with gabapentin, pregabalin or duloxetine enter as independent variables. The level of statistical significance is set at $\alpha=0.05$.

\section{Standard summary statistics}

Continuous variables will be summarised with descriptive statistics by displaying sample size, mean, median, minimum, maximum, SD and $95 \%$ CI. Categorial data will be summarised using frequencies and percentages.

\section{Analysis populations}

The primary analysis will be performed in the intentionto-treat (ITT) population. Missing values will be imputed by multiple imputation technique. ITT population is defined as all participants having at least one dose of treatment and at least one measurement of the primary endpoint under treatment. Per-protocol (PP) analysis will also be performed. PP population is defined as all participants, valid for ITT analysis not fulfilling any of the following PP-analysis exclusion criteria: Major protocol deviation, compliance to $\mathrm{IMP}<80 \%$, or missing data. Further exclusion criteria may be defined during blind data review.

The safety analysis set will include all participants who received at least one dose of treatment. Parameters for safety analysis will include adverse events, concomitant medication, laboratory safety variables, physical examination, vital signs. 


\section{Subgroup analyses}

Exploratory subgroup analyses may be performed, for example, for participants taking the IMP as add-on therapy, and those who had no parallel treatment. Furthermore, exploratory subgroup analyses are planned to observe the influence of the COVID-19 pandemic, for example, for participants who were exposed to or infected with SARSCoV-2. Details about subgroup analyses will be defined in the statistical analysis plan.

\section{Exploratory analyses}

Exploratory analyses will assess dose and time effects by using appropriate statistical models. Especially, analyses in the time course will adjust for the fact (by, eg, mixed models or covariance pattern analyses), that measurements are repeatedly taken in the same participant.

\section{Health economic evaluation}

The health economic evaluation will be considered from the perspective of the German statutory health insurance. A cost-effectiveness analysis will be performed in terms of additional costs per change in PGIC as well as a cost-utility analysis to calculate the additional costs required for an improvement in quality-adjusted life-years (QALYs). The QALYs are based on a health-related quality of life, measured by the EQ-5D-5L, and evaluated by a German tariff to generate utilities. The $95 \%$ CIs will be obtained parametrically for the incremental cost-effectiveness ratio (ICER) and incremental cost-utility ratio (ICUR) and non-parametrically by a bootstrap procedure. Univariate and probabilistic sensitivity analyses will be performed to estimate the robustness of the ICER/ICUR. ${ }^{49}$

\section{ETHICS AND DISSEMINATION}

The trial received favourable opinion by the Ethics Committee at the Faculty of Medicine of Heinrich Heine University, Düsseldorf, Germany on 27 July 2018 and was approved by the German Federal Institute for Drugs and Medical Devices on 8 June 2018.

An electronic data capture system according to the guideline for good clinical practice (GCP) by the International Council on Harmonisation of Technical Requirements for Registration of Pharmaceuticals for Human Use (ICH) guidelines will be used. A blind data review meeting will be performed before database lock and unblinding in the presence of sponsor.

\section{Benefits and harms}

In clinical trials, no severe adverse events caused by benfotiamine were observed, except for one case of sudden cardiac death (EudraCT: 2013-001058-85) which was reported by the investigator as a suspected unexpected serious adverse reaction possibly related to the IMP. All other adverse events observed were usually only mild and did not require drug withdrawal. The expected rate of adverse events during benfotiamine treatment is $5 \%-10 \%$ of participants as a maximum, mainly gastrointestinal disorders or hypersensitivity reactions.

The recommended daily dose of benfotiamine for the treatment of neuropathies is $300 \mathrm{mg}$, but doses as high as $900 \mathrm{mg}$ /day were delivered for 3 months, ${ }^{19}$ while doses of $300 \mathrm{mg} /$ day were administered for 2 years. ${ }^{14}$ In the BENDIP study, ${ }^{50}$ a benfotiamine dose of $300 \mathrm{mg}$ two times a day $(600 \mathrm{mg} /$ day $)$, but not $300 \mathrm{mg}$ four times a day $(1200 \mathrm{mg} /$ day $)$ was associated with an improvement of neuropathic symptoms after 5 weeks. Therefore, to ensure adequate dosing of benfotiamine throughout the entire trial period, a fixed dose of benfotiamine $300 \mathrm{mg}$ two times a day over 12 months was chosen.

There are no reports on teratogenic effects or adverse effects on development of the fetus, nor negative results on genotoxicity or carcinogenicity. No vulnerable groups of patients have been identified. Due to the extremely low toxicity of thiamine, different expert groups to date could not define adverse effect levels or tolerable upper intake level.

In clinical studies, isolated cases of gastrointestinal disturbances like nausea or other disorders have been reported. Allergic/hypersensitivity reactions occurred preferably after parenteral application and only rarely after oral intake. A causal relationship to vitamin $B_{1}$ as well as a possible dose relation are not fully understood. However, it has to be taken into account, that some observations from clinical trials are based on combination therapy with other B-vitamins. Therefore, the risk related to the IMP administration is low in this trial with the dose chosen.

Skin biopsy is a minimally invasive validated method widely used in clinical trials and practice, the risks of which are relatively low (intolerance to local anaesthetics, local bleeding, infection). Patients with diabetes might have a higher risk of impaired wound healing. Other conditions that may impair wound healing are excluded from the trial.

On the other hand, DSPN is associated with considerable morbidity and carries an increased risk of mortality. Since a worldwide-approved disease-modifying pharmacotherapy is not available, there is an unmet medical need for such a treatment, which could delay the progression of DSPN or even induce its regression to ultimately prevent the development of feared sequelae of DSPN such as diabetic foot ulcers and amputations. Therefore, the potential benefits of the present trial outweigh the possible risks.

\section{Ancillary and post-trial care}

No post-trial treatment is planned. The investigator will monitor participants with adverse and severe adverse events, until the event has resolved or stabilised and any abnormal laboratory values have returned to baseline; or until there is a satisfactory explanation for the changes observed. 


\section{Monitoring, audits and inspections}

Monitoring and audits will be performed by Wörwag Pharma \& Co. KG, Böblingen, Germany, in accordance to ICH GCP E6(R2) 5.18. The investigators and trial site agree, by participating in the trial, to allow and cooperate with audits and inspections.

\section{Dissemination policy}

Trial results will be published in in the clinical trial register, in scientific publications in peer-reviewed journals, conference abstracts, and via online and print media.

\section{Author affiliations}

${ }^{1}$ Institute for Clinical Diabetology, German Diabetes Center, Leibniz Center for Diabetes Research at Heinrich Heine University, Düsseldorf, Germany ${ }^{2}$ Department of Endocrinology and Diabetology, Medical Faculty and University Hospital Düsseldorf, Heinrich Heine University, Düsseldorf, Germany ${ }^{3}$ German Center for Diabetes Research, Partner Düsseldorf, Munich-Neuherberg, Germany

${ }^{4}$ Institute for Biometrics and Epidemiology, German Diabetes Center, Leibniz Center for Diabetes Research at Heinrich Heine University, Düsseldorf, Germany ${ }^{5}$ Institute for Clinical Biochemistry and Pathobiochemistry, German Diabetes Center, Leibniz Center for Diabetes Research at Heinrich Heine University, Düsseldorf, Germany

${ }^{6}$ Wörwag Pharma GmbH und Co KG, Böblingen, Germany

${ }^{7}$ Institute for Health Services Research and Health Economics, German Diabetes Center, Leibniz Center for Diabetes Research at Heinrich Heine University, Düsseldorf, Germany

${ }^{8}$ Institute for Health Services Research and Health Economics, Centre for Health and Society, Medical Faculty and University Hospital Düsseldorf at Heinrich-HeineUniversity, Düsseldorf, Germany

${ }^{9}$ Centre for Health and Society, Medical Faculty and University Hospital Düsseldorf, Heinrich-Heine-University, Düsseldorf, Germany

Contributors DZ, GJB, CR and J-CW conceived and refined the trial design. DZ, AS, CH, BK, Al, HA-H, CR and MR compiled the protocol for assessments. KS and OK developed the statistical analysis plan. GJB and GS drafted the manuscript. All authors critically contributed to the writing and approved the final manuscript for publication.

Funding The trial is sponsored by Wörwag Pharma \& Co. KG, Calwer Straße 7, 71 034 Böblingen, Germany (grant number N/A).

Competing interests DZ and GJB received honoraria from Wörwag Pharma for speaking and consulting activities. $\mathrm{CH}$ received a research grant from SanofiAventis. CR and J-CW are employees of Wörwag Pharma.

Patient consent for publication Not applicable.

Provenance and peer review Not commissioned; externally peer reviewed.

Supplemental material This content has been supplied by the author(s). It has not been vetted by BMJ Publishing Group Limited (BMJ) and may not have been peer-reviewed. Any opinions or recommendations discussed are solely those of the author(s) and are not endorsed by BMJ. BMJ disclaims all liability and responsibility arising from any reliance placed on the content. Where the content includes any translated material, BMJ does not warrant the accuracy and reliability of the translations (including but not limited to local regulations, clinical guidelines, terminology, drug names and drug dosages), and is not responsible for any error and/or omissions arising from translation and adaptation or otherwise.

Open access This is an open access article distributed in accordance with the Creative Commons Attribution Non Commercial (CC BY-NC 4.0) license, which permits others to distribute, remix, adapt, build upon this work non-commercially, and license their derivative works on different terms, provided the original work is properly cited, appropriate credit is given, any changes made indicated, and the use is non-commercial. See: http://creativecommons.org/licenses/by-nc/4.0/.

\section{ORCID iD}

Gidon J Bönhof http://orcid.org/0000-0003-1446-6592
REFERENCES

1 Bönhof GJ, Herder C, Strom A, et al. Emerging biomarkers, tools, and treatments for diabetic polyneuropathy. Endocr Rev 2019;40:153-92.

2 Pop-Busui R, Boulton AJM, Feldman EL, et al. Diabetic neuropathy: a position statement by the American diabetes association. Diabetes Care 2017;40:136-54.

3 Jensen TS, Karlsson P, Gylfadottir SS, et al. Painful and non-painful diabetic neuropathy, diagnostic challenges and implications for future management. Brain 2021;144:1632-45.

4 Bönhof GJ, Herder C, Ziegler D. Diagnostic tools, biomarkers, and treatments in diabetic polyneuropathy and cardiovascular autonomic neuropathy. Curr Diabetes Rev 2021. doi:10.2174/15733998176662 10412123740. [Epub ahead of print: 12 Apr 2021] (published Online First: 2021/04/14).

5 Sloan G, Selvarajah D, Tesfaye S, Pathogenesis TS. Pathogenesis, diagnosis and clinical management of diabetic sensorimotor peripheral neuropathy. Nat Rev Endocrinol 2021;17:400-20.

6 Hammes H-P, Du X, Edelstein D, et al. Benfotiamine blocks three major pathways of hyperglycemic damage and prevents experimental diabetic retinopathy. Nat Med 2003;9:294-9.

7 Berrone E, Beltramo E, Solimine C, et al. Regulation of intracellular glucose and polyol pathway by thiamine and benfotiamine in vascular cells cultured in high glucose. $\mathrm{J} \mathrm{Biol} \mathrm{Chem}$ 2006;281:9307-13.

8 Schmid U, Stopper $\mathrm{H}$, Heidland A, et al. Benfotiamine exhibits direct antioxidative capacity and prevents induction of DNA damage in vitro. Diabetes Metab Res Rev 2008;24:371-7.

9 Bozic I, Savic D, Stevanovic I, et al. Benfotiamine upregulates antioxidative system in activated BV-2 microglia cells. Front Cell Neurosci 2015;9:351.

10 Stracke H, Hammes HP, Werkmann D, et al. Efficacy of benfotiamine versus thiamine on function and glycation products of peripheral nerves in diabetic rats. Exp Clin Endocrinol Diabetes 2001;109:330-6.

11 Stracke H, Lindemann A, Federlin K. A benfotiamine-vitamin B combination in treatment of diabetic polyneuropathy. Exp Clin Endocrinol Diabetes 1996;104:311-6.

12 Haupt E, Ledermann H, Köpcke W. Benfotiamine in the treatment of diabetic polyneuropathy--a three-week randomized, controlled pilot study (BEDIP study). Int J Clin Pharmacol Ther 2005;43:71-7.

13 Winkler G, Pál B, Nagybéganyi E, et al. Effectiveness of different benfotiamine dosage regimens in the treatment of painful diabetic neuropathy. Arzneimittelforschung 1999;49:220-4.

14 Fraser DA, Diep LM, Hovden IA. The effects of long-term oral benfotiamine supplementation on peripheral nerve function and inflammatory markers in patients with type 1 diabetes: a 24-month, double-blind, randomized, placebo-controlled trial. Diabetes Care 2012;35:1095-7.

15 Fraser DA, Diep LM, Hovden IA, et al. The effects of long-term oral benfotiamine supplementation on peripheral nerve function and inflammatory markers in patients with type 1 diabetes: a 24-month, double-blind, randomized, placebo-controlled trial. Diabetes CareDiabetes Care 2012;35:1095-7.

16 Stirban OA, Zeller-Stefan H, Schumacher J, et al. Treatment with benfotiamine in patients with diabetic sensorimotor polyneuropathy: A double-blind, randomized, placebocontrolled, parallel group pilot study over 12 months. J Diabetes Complications 2020;34:107757.

17 Stirban A, Negrean M, Stratmann B, et al. Benfotiamine prevents macro- and microvascular endothelial dysfunction and oxidative stress following a meal rich in advanced glycation end products in individuals with type 2 diabetes. Diabetes Care 2006;29:2064-71.

18 Stirban A, Nandrean S, Kirana S, et al. Benfotiamine counteracts smoking-induced vascular dysfunction in healthy smokers. Int $J$ Vasc Med 2012;2012:968761.

19 Alkhalaf A, Klooster A, van Oeveren W, et al. A double-blind, randomized, placebo-controlled clinical trial on benfotiamine treatment in patients with diabetic nephropathy. Diabetes Care 2010;33:1598-601.

20 Perkins BA, Lovblom LE, Lewis EJH, et al. Corneal confocal microscopy predicts the development of diabetic neuropathy: a longitudinal diagnostic multinational Consortium study. Diabetes Care 2021;44:dc210476

21 Herder C, Roden M, Ziegler D. Novel insights into sensorimotor and cardiovascular autonomic neuropathy from recent-onset diabetes and population-based cohorts. Trends Endocrinol Metab 2019;30:286-98.

22 Haque F, Reaz MBI, Ali SHM, et al. Performance analysis of noninvasive electrophysiological methods for the assessment of diabetic sensorimotor polyneuropathy in clinical research: a 
systematic review and meta-analysis with trial sequential analysis. Sci Rep 2020;10:21770.

23 Papanas N, Ziegler D. Corneal confocal microscopy: recent progress in the evaluation of diabetic neuropathy. J Diabetes Investig 2015;6:381-9.

24 Azmi S, Jeziorska M, Ferdousi M, et al. Early nerve fibre regeneration in individuals with type 1 diabetes after simultaneous pancreas and kidney transplantation. Diabetologia 2019;62:1478-87.

25 Püttgen S, Bönhof GJ, Strom A, et al. Augmented corneal nerve fiber branching in painful compared with painless diabetic neuropathy. $J$ Clin Endocrinol Metab 2019;104:6220-8.

26 Lauria G, Hsieh ST, Johansson O, et al. European Federation of neurological Societies/Peripheral nerve Society guideline on the use of skin biopsy in the diagnosis of small fiber neuropathy. Report of a joint Task force of the European Federation of neurological societies and the peripheral nerve Society. Eur J Neurol 2010;17:903-49.

27 Lauria G, Bakkers M, Schmitz C, et al. Intraepidermal nerve fiber density at the distal leg: a worldwide normative reference study. $J$ Peripher Nerv Syst 2010;15:202-7.

28 Tesfaye S, Boulton AJM, Dyck PJ, et al. Diabetic neuropathies: update on definitions, diagnostic criteria, estimation of severity, and treatments. Diabetes Care 2010;33:2285-93.

29 Smith AG, Russell J, Feldman EL, et al. Lifestyle intervention for prediabetic neuropathy. Diabetes Care 2006;29:1294-9.

30 Singleton JR, Marcus RL, Jackson JE, et al. Exercise increases cutaneous nerve density in diabetic patients without neuropathy. Ann Clin Transl Neurol 2014;1:844-9.

31 Kluding PM, Pasnoor M, Singh R, et al. The effect of exercise on neuropathic symptoms, nerve function, and cutaneous innervation in people with diabetic peripheral neuropathy. J Diabetes Complications 2012;26:424-9.

32 Nowotny K, Jung T, Höhn A, et al. Advanced glycation end products and oxidative stress in type 2 diabetes mellitus. Biomolecules 2015;5:194-222.

33 Ziegler D, Papanas N, Zhivov A, et al. Early detection of nerve fiber loss by corneal confocal microscopy and skin biopsy in recently diagnosed type 2 diabetes. Diabetes 2014;63:2454-63.

34 Strom A, Brüggemann J, Ziegler I, et al. Pronounced reduction of cutaneous Langerhans cell density in recently diagnosed type 2 diabetes. Diabetes 2014;63:1148-53.

35 Ziegler D, Strom A, Brüggemann J, et al. Overexpression of cutaneous mitochondrial superoxide dismutase in recent-onset type 2 diabetes. Diabetologia 2015;58:1621-5.

36 Bönhof GJ, Strom A, Püttgen S, et al. Patterns of cutaneous nerve fibre loss and regeneration in type 2 diabetes with painful and painless polyneuropathy. Diabetologia 2017;60:2495-503.

37 Spallone V. Update on the impact, diagnosis and management of cardiovascular autonomic neuropathy in diabetes: what is defined, what is new, and what is unmet. Diabetes Metab J 2019;43:3-30.
38 Kück J-L, Bönhof GJ, Strom A, et al. Impairment in baroreflex sensitivity in recent-onset type 2 diabetes without progression over 5 years. Diabetes 2020;69:1011-9.

39 Beckert S, Witte MB, Königsrainer A, et al. The impact of the microlightguide $\mathrm{O} 2 \mathrm{C}$ for the quantification of tissue ischemia in diabetic foot ulcers. Diabetes Care 2004;27:2863-7.

40 Yamamoto-Suganuma R, Aso Y. Relationship between postocclusive forearm skin reactive hyperaemia and vascular disease in patients with Type 2 diabetes--a novel index for detecting micro- and macrovascular dysfunction using laser Doppler flowmetry. Diabet Med 2009;26:83-8.

41 Bril V, Tomioka S, Buchanan RA, et al. Reliability and validity of the modified Toronto clinical neuropathy score in diabetic sensorimotor polyneuropathy. Diabet Med 2009;26:240-6.

42 Herder C, Kannenberg JM, Huth C, et al. Proinflammatory cytokines predict the incidence and progression of distal sensorimotor polyneuropathy: KorA F4/FF4 study. Diabetes Care 2017;40:569-76.

43 Herder C, Kannenberg JM, Carstensen-Kirberg M, et al. A systemic inflammatory signature reflecting cross talk between innate and adaptive immunity is associated with incident polyneuropathy: KorA F4/FF4 study. Diabetes 2018;67:2434-42.

44 Ziegler D, Schleicher E, Strom A, et al. Association of transketolase polymorphisms with measures of polyneuropathy in patients with recently diagnosed diabetes. Diabetes Metab Res Rev 2017;33. doi:10.1002/dmrr.2811. [Epub ahead of print: 1505 2016].

45 Hoppel F, Garcia-Souza LF, Kantner-Rumplmair W, et al. Human platelet mitochondrial function reflects systemic mitochondrial alterations: a protocol for application in field studies. Cells 2021;10:2088.

46 Larsen S, Nielsen J, Hansen CN, et al. Biomarkers of mitochondrial content in skeletal muscle of healthy young human subjects. $J$ Physiol 2012;590:3349-60.

47 Strom A, Kaul K, Brüggemann J, et al. Lower serum extracellular superoxide dismutase levels are associated with polyneuropathy in recent-onset diabetes. Exp Mol Med 2017;49:e394.

48 Tavakoli M, Mitu-Pretorian M, Petropoulos IN, et al. Corneal confocal microscopy detects early nerve regeneration in diabetic neuropathy after simultaneous pancreas and kidney transplantation. Diabetes 2013;62:254-60.

49 Mortsiefer A, Wilm S, Santos S, et al. Family conferences and shared prioritisation to improve patient safety in the frail elderly (COFRAIL): study protocol of a cluster randomised intervention trial in primary care. Trials 2020;21:285.

50 Stracke H, Gaus W, Achenbach U, et al. Benfotiamine in diabetic polyneuropathy (BENDIP): results of a randomised, double blind, placebo-controlled clinical study. Exp Clin Endocrinol Diabetes 2008;116:600-5. 